\title{
Understanding the white-light flare on 2012 March 9: evidence of a two-step magnetic reconnection
}

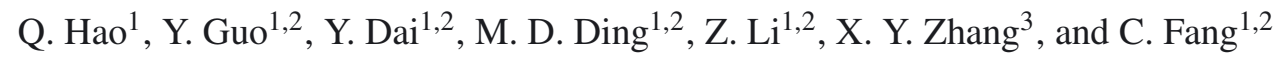 \\ ${ }^{1}$ School of Astronomy and Space Science, Nanjing University, 210093 Nanjing, PR China \\ e-mail: fangc@nju.edu.cn \\ 2 Key Laboratory of Modern Astronomy and Astrophysics (Nanjing University), Ministry of Education, 210093 Nanjing, PR China \\ 3 Yunnan Astronomical Observatory, Chinese Academy of Sciences, 620011 Kunming, PR China \\ e-mail: xyz@ynao.ac.cn
}

Received 3 July 2012 / Accepted 23 July 2012

\section{ABSTRACT}

\begin{abstract}
Aims. We attempt to understand the white-light flare (WLF) that was observed on 2012 March 9 with a newly constructed multiwavelength solar telescope called the Optical and Near-infrared Solar Eruption Tracer (ONSET).

Methods. We analyzed WLF observations in radio, $\mathrm{H} \alpha$, white-light, ultraviolet, and X-ray bands. We also studied the magnetic configuration of the flare via the nonlinear force-free field (NLFFF) extrapolation and the vector magnetic field observed by the Helioseismic and Magnetic Imager (HMI) on board the Solar Dynamics Observatory (SDO).

Results. Continuum emission enhancement clearly appeared at the $3600 \AA$ and $4250 \AA$ bands, with peak contrasts of $25 \%$ and $12 \%$, respectively. The continuum emission enhancement closely coincided with the impulsive increase in the hard X-ray emission and a microwave type III burst at 03:40 UT. We find that the WLF appeared at one end of either the sheared or twisted field lines or both. There was also a long-lasting phase in the $\mathrm{H} \alpha$ and soft X-ray bands after the white-light emission peak. In particular, a second, yet stronger, peak appeared at 03:56 UT in the microwave band.

Conclusions. This event shows clear evidence that the white-light emission was caused by energetic particles bombarding the lower solar atmosphere. A two-step magnetic reconnection scenario is proposed to explain the entire process of flare evolution, i.e., the firststep magnetic reconnection between the field lines that are highly sheared or twisted or both, and the second-step one in the current sheet, which is stretched by the erupting flux rope. The WLF is supposed to be triggered in the first-step magnetic reconnection at a relatively low altitude.
\end{abstract}

Key words. magnetic reconnection - Sun: flares - Sun: magnetic topology - Sun: radio radiation

\section{Introduction}

White-light flares (WLFs) are flares that have emission visible in the optical continuum (Neidig et al. 1993). Research on WLFs can provide critical insight into the possible energy transport and heating mechanisms in the lower solar atmosphere (Neidig 1989; Ding et al. 1999). The first WLF was observed 153 years ago (Carrington 1859). However, when including the first observations of WLFs from space (Hudson et al. 1992), up to now fewer than $150 \mathrm{WLFs}$ have been reported in the literature. These are thought to be rare and among the most energetic flaring events. In most cases, enhanced continuum emission is detected in the Balmer and Paschen continuum. In a few cases, however, enhanced emission in the infrared continuum was also reported (Liu et al. 2001; Xu et al. 2004).

Hudson et al. (2006) used the white-light (WL) channel $(>1500 \AA)$ on board the Transition Region and Coronal Explorer (TRACE; Handy et al. 1999) with a spatial resolution of $1^{\prime \prime}$ and detected WL emission for flares down to GOES class C1.6. They suggested that the WL continuum may occur in all flares. Fletcher et al. (2007) also analyzed nine flares observed by TRACE in WL. However, because the TRACE WL/ultraviolet (UV) emission always has a higher contrast than that of the traditional WLFs and the observations are sometimes saturated, it is unclear whether the TRACE continuum is affected by UV emission. Jess et al. (2008) used high resolution observations of the one-meter Swedish Solar Telescope to detect WL emission in the blue continuum around $3954 \AA$ with a peak intensity $300 \%$ above the quiescent flux in a C2.0 flare. This high emission contrast, although restricted to a small area, is surprising and it remains to be verified whether it is a common feature in WLF observations.

Using Yohkoh observations, Matthews et al. (2003) reported 28 WLFs in the G-band and found that they have a strong association with hard X-ray emission. Wang (2009) studied 13 flares observed by Hinode (Tsuneta et al. 2008) and found that there is a correlation between the GOES soft X-ray flux and the flare emission in the G-band. However, as the author pointed out, since Hinode G-band observations are contaminated by $\mathrm{CH}$ band emission and the time resolution of the observations is insufficiently high, it is unclear whether the peak values and the cut-off visibility of WLFs are real.

Can the origin of WLFs be explained by heating and energy transportation in the lower solar atmosphere, even around the minimum temperature region? Several mechanisms have been proposed, including electron beam bombardment followed by radiative backwarming (Machado et al. 1989; Metcalf et al. 1990), energy transportation by Alfvén waves (Fletcher \& Hudson 2008), magnetic reconnection in the lower atmosphere (Ding et al. 1999; Chen et al. 2001), heating by chromospheric 
condensation (Gan et al. 1992) and so on. It is necessary to distinguish between two types of WLFs (Machado et al. 1986). Fang \& Ding (1995) studied the different characteristics of each one using both observations and atmospheric non-localthermodynamic-equilibrium modeling. In the case of type I WLFs, there exists a strong time correlation between the peak of either the hard X-ray emission or the microwave burst and the maximum of the continuum emission, there is a strong Balmer jump in the spectra, and the Balmer lines, particularly the $\mathrm{H} \alpha$ line, are usually strong and broad. However, type II WLFs do not display the above features. For type I WLFs, either the electron bombardment or the following backwarming or both would be plausible mechanisms (Ding et al. 2003; Chen \& Ding 2005; Fletcher et al. 2007); however, for type II WLFs, the mechanism is more likely to be magnetic reconnection in the lower atmosphere (Chen et al. 2001; Jiang et al. 2010).

In this letter, we report a WLF observed in radio, $\mathrm{H} \alpha$, WL, ultraviolet, and X-ray bands. We study in particular the magnetic configuration of the flare via the nonlinear force-free field (NLFFF) extrapolation and the vector magnetic field observed by the Helioseismic and Magnetic Imager (HMI) on board the Solar Dynamics Observatory (SDO). Our data analysis and results are presented in Sect. 2, and both our discussion and conclusions are given in Sect. 3.

\section{Data analysis and results}

An M6.3 two-ribbon flare was observed at N15W01 in active region NOAA 11429 on 2012 March 9 by the Optical and Nearinfrared Solar Eruption Tracer (ONSET), which was constructed by Nanjing University in cooperation with Yunnan Astronomical Observatory (Fang et al. 2012). The telescope ONSET is installed at a new solar observing site (E102.57, $\left.\mathrm{N} 24.38^{\circ}\right)$ near the Fuxian Lake, $60 \mathrm{~km}$ far from Kunming. The seeing is both stable and good (Liu \& Beckers 2001).

The ONSET consists of four tubes: (1) a near-infrared vacuum tube, with an aperture of $27.5 \mathrm{~cm}$, operating at the He I line center and wings up to $10830 \pm 2.5 \AA$ with a full width at half maximum (FWHM) of $0.25 \AA$; (2) a chromospheric (H $\alpha$ ) vacuum tube, with an aperture of $27.5 \mathrm{~cm}$, working at the $\mathrm{H} \alpha$ line center and wings up to $6562.8 \pm 1.5 \AA$ with an FWHM of $0.25 \AA$; (3) a WL vacuum tube, with an aperture of $20 \mathrm{~cm}$, working in either the $3600 \AA$ or $4250 \AA$ continuum with an FWHM of $15 \AA$; and (4) a guiding tube.

The observations were made from about 03:00 UT to 05:00 UT at $3600 \AA, 4250 \AA, \mathrm{H} \alpha$ line center, and H $\alpha 6562.8 \pm$ $0.5 \AA$, with a cadence of $1 \mathrm{~min}$. The pixel size is about $1^{\prime \prime}$. We also analyzed UV data at $1700 \AA$ and $1600 \AA$, which were observed by the Atmospheric Imaging Assembly (AIA; Lemen et al. 2012) on board SDO. Figure 1 depicts the images at $3600 \AA, 1700 \AA$, and the $\mathrm{H} \alpha$ line center before the WLF (03:25 UT), at the peak time of the WLF (03:40 UT), and after the WLF (03:55 UT). The WL emission can be clearly seen in both the $3600 \AA$ and $4250 \AA$ continua at 03:40 UT. The size of the WLF is about $3^{\prime \prime} \times 3^{\prime \prime}$ with a duration of about 2-3 min. However, even before the WLF peak time, the emission at both H $\alpha$ and $1700 \AA$ already appeared as bright threads at 03:25 UT. As seen in Fig. 1, these threads were very close to the polarity inversion line separating the two highly sheared ribbons. The image at $1600 \AA$ is similar to that at $1700 \AA$. After the WLF peak, the two flare ribbons seen in $1700 \AA$ and $\mathrm{H} \alpha$ bands are separated more widely than before, which indicates that the magnetic reconnection occurred continuously.

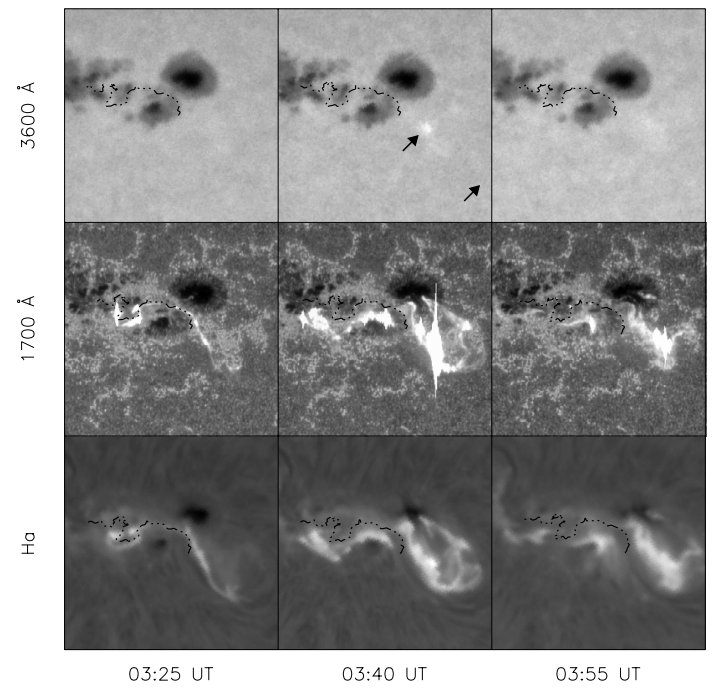

Fig. 1. Images showing the M6.3 flare evolution at $3600 \AA, 1700 \AA$, and the $\mathrm{H} \alpha$ line center before the WLF (left column), at the peak time of the WLF (middle column), and after the WLF (right column) on 2012 March 9. The field of view of each image is $200^{\prime \prime} \times 200^{\prime \prime}$. The location of the center of the field of view is around $\left(30^{\prime \prime}, 380^{\prime \prime}\right)$. The upper-left arrow indicates the area where the white-light and $\mathrm{H} \alpha$ intensities are measured as shown in Fig. 2. The lower-right arrow indicates the quiet region selected as the background. Dash-dotted lines mark the polarity inversion line of the line-of-sight magnetogram.

Figure 2 depicts the light curves of the WLF in different wavebands. We note that for (1) $\mathrm{H} \alpha, R=I / I_{0}$ and (2) both the $4250 \AA$ and $3600 \AA$ bands, the contrast is defined as $C=$ $\left(I-I_{0}\right) / I_{0}$, where $I_{0}$ is the intensity of the nearby quiet region. It can be seen that the peaks in different wavebands (except for the soft X-ray) are precisely consistent at around 03:40 UT, which suggests that there is a close relationship between the energetic particles and the WL emission. The microwave burst data were recorded by the Solar Radio Broadband Spectrometer (SRBS; Fu et al. 2004) at the Huairou Station of the National Astronomical Observatory of China. Near 03:40 UT, there is a type III burst with a positive frequency drift of $700 \mathrm{MHz} \mathrm{s}^{-1}$ (Liu, priv. comm.), which indicates that an electron beam bombarded the lower solar atmosphere from the upper atmosphere, which is supposed to be related to the observed WLF. In addition to the peak at around 03:40 UT, there is another series of peaks in the $3.79 \mathrm{GHz}$ band, especially the main peak at 03:56 UT as shown in Fig. 2a. We checked other observations, such as extreme-ultraviolet (EUV) images, and found that there was enhanced emission only in the active region NOAA 11429 from 03:40 UT to 03:56 UT. Therefore, the main peak at $3.79 \mathrm{GHz}$ implies that there were still energetic particles at 03:56 UT. Figure $2 \mathrm{~b}$ shows that the $\mathrm{H} \alpha$ line was strong and broad both during the WLF peak and after it, since both the line center and line wing display strong emission. Figure $2 \mathrm{c}$ shows that the contrasts at the WLF peak time in the $3600 \AA$ and $4250 \AA$ continua are about $25 \%$ and $12 \%$, respectively. The peak time of the soft $\mathrm{X}$-ray flux is about 03:53 UT, which is approximately $13 \mathrm{~min}$ later than the hard X-ray peak time. This behavior is due to the Neupert effect, i.e., the thermal fluxes coinciding with the integral of the non-thermal fluxes. However, the 13 min delay is relatively long (Li \& Gan 2006) and the soft X-ray does not drop too much even 30 min after the hard X-ray peak. This strongly suggests that the flare continuously released energy in phase after the WLF peak. 


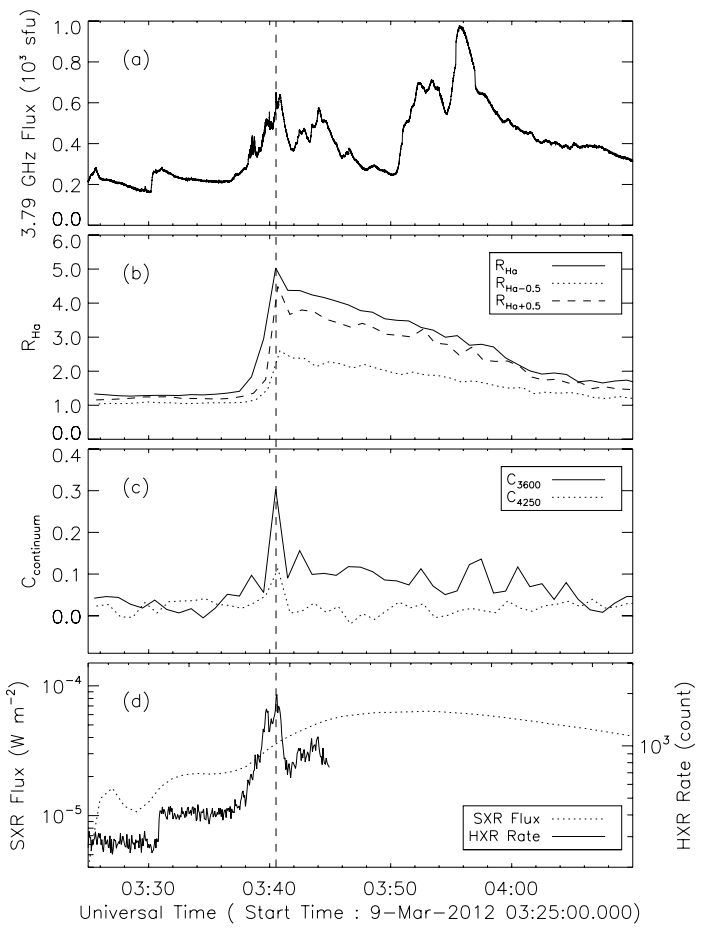

Fig. 2. Time profiles of the radio flux at $3.79 \mathrm{GHz}$, the intensity enhancement $R=I / I_{0}$ at the $\mathrm{H} \alpha$ line center and $6562.8 \pm 0.5 \AA$, the continuum contrast $C=\left(I-I_{0}\right) / I$ at $4250 \AA$ and $3600 \AA$, the RHESSI hard X-ray (50-100 keV) flux, and the GOES soft X-ray (1-8 $\AA$ ) flux of the WLF. The light curves in the white-light and $\mathrm{H} \alpha$ bands are computed in the WLF kernel as shown in Fig. 1, and those in other bands are computed for the entire solar disk. The HXR data after 03:45 UT are omitted owing to a change of the various attenuators.

Figure $3 \mathrm{a}$ shows the vector magnetic field observed by SDO/HMI at 03:34 UT, which was 6 min before the WLF peak at $03: 40$ UT. The $180^{\circ}$ ambiguity is removed by the minimum energy method (Leka et al. 2009). We first pre-process the vector magnetic field data to remove the magnetic force and torque (Wiegelmann et al. 2006). We then correct for projection effects and transform the line-of-sight and transverse components of the vector magnetic field to the heliographic components as described in Gary \& Hagyard (1990). Finally, an NLFFF extrapolation is made to obtain the magnetic field configuration with the optimization method (Wheatland et al. 2000; Wiegelmann 2004). Figure $3 b$ depicts the magnetic field lines and the $3600 \AA$ contours. Highly sheared field lines with relatively low altitudes close to the polarity inversion line are clearly present below a set of higher magnetic arcades. Some field lines in the core field region are twisted around each other. The WLF is located at the western end of the sheared or twisted or both magnetic field lines, where the magnetic field is relatively weak.

\section{Discussion and conclusions}

A WLF was clearly observed on 2012 March 9 in different wavebands, particularly in the $3600 \AA$ and $4250 \AA$ continuum bands by the new telescope ONSET. Figure 2 clearly shows that the peak times of the emissions at $3.79 \mathrm{GHz}$, the $\mathrm{H} \alpha$ line center, H $\alpha 6562.8 \pm 0.5 \AA, 3600 \AA, 4250 \AA$, and the hard X-ray are temporally coincident. The contrast at $3600 \AA$ is larger than that at $4250 \AA$, implying that the WLF has a Balmer jump. These demonstrate that it is a type I WLF (Fang \& Ding 1995).
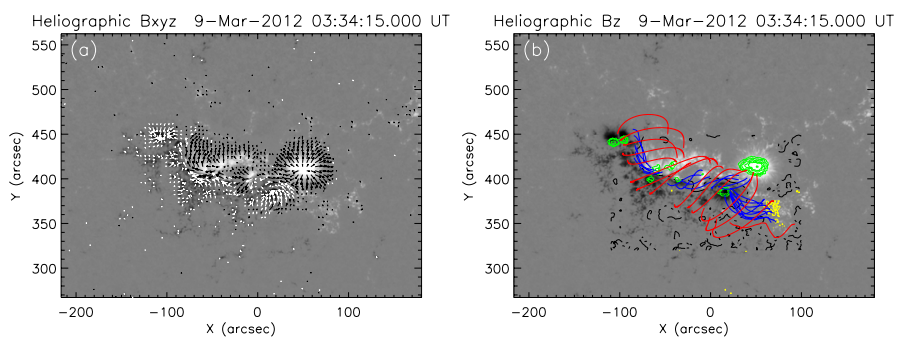

Fig. 3. a) Projection-corrected vector magnetic field, where arrows represent the horizontal components. b) Radial magnetic field overlaid on the $3600 \AA$ contour (yellow and green lines), the polarity inversion line (dash-dotted lines), and the NLFFF field lines (blue and red lines). The magnetic field has been corrected for projection effects. The yellow lines indicate the location of the WLF, while the green ones indicate the sunspot place.

However, the general configurations in $\mathrm{H} \alpha$ and $1700 \AA$ are quite different from that of the WLF: the first two have a ribbon-like structure, while the last has a point-like one, though both the $\mathrm{H} \alpha$ and $1700 \AA$ images were relatively bright at both the WLF time and location. This implies that what we observed at either $1700 \AA$ or in the $\mathrm{H} \alpha$ wing cannot represent the WL emission feature.

Using the vector magnetic field data from SDO/HMI, we make an NLFFF extrapolation to obtain the magnetic field configuration around the WLF site and before the WLF peak time. Figure 3 shows that the WLF occurred at one end of the highly sheared or twisted field lines, which suggests that magnetic reconnection occurred in the low-lying core field region. Otherwise, the energetic particles produced by the magnetic reconnection cannot have precipitated to the footpoint of the core field lines. A similar situation had been previously observed by Guo et al. (2012), who found that hard X-ray sources appear at the footpoints of an erupting flux rope. Considering a microwave type III burst and hard X-ray burst occurred all at the WLF time, we suggest that an electron beam has accelerated and bombarded the lower atmosphere, producing the WLF through collisional heating or backwarming. The WLF only appeared in the region with positive polarity, because the magnetic field there was relatively weak compared to the region of conjugate polarity connected by the field line.

Figure 4 depicts a cartoon to show the entire mechanism and illustrate our two-step magnetic reconnection scenario, i.e., magnetic reconnection between the highly sheared or twisted field lines and that in the current sheet stretched by the erupting flux rope. Before the magnetic reconnection and the magnetic-flux rope eruption, the whole magnetic field configuration is constituted by a core field with highly sheared or twisted field lines and a potential envelope field before the flare onset as shown in Fig. 4a. The core field has an elbow curved that is in the opposite direction at each end, which extends out of the envelope field.

For the first-step magnetic reconnection, the core field first started to reconnect as shown in Fig. 4b, which corresponds to the $1700 \AA$ and $\mathrm{H} \alpha$ observations at 03:25 UT. The $1700 \AA$ emission flashed at the center of the core field region, and a helioseismic wave also appeared in the same region (Zhao, priv. comm.). Then, the inner-core field lines continued to reconnect with the outer, curved elbow field-lines. The core field lines finally formed an erupting flux rope and the WLF appeared on the north side at the footpoints of the reconnected field lines below the reconnection site at 03:40 UT, as shown in Fig. 4c. The microwave peak at around 03:40 UT is lower than that at 


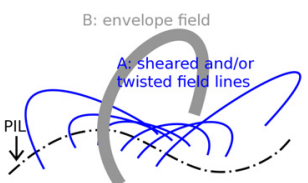

(a) Before Eruption

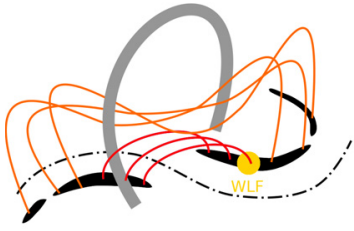

(c) White-Light Flare Peak

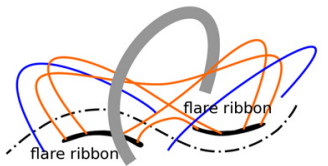

(b) Eruption Onset

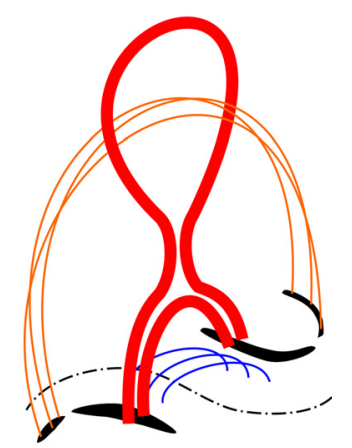

(d) Soft X-ray Flare Peak
Fig. 4. A cartoon showing the two-step magnetic reconnection scenario, i.e., the first step between the highly sheared or twisted field lines and the second in the current sheet stretched by the erupting flux rope. Gray, blue, orange, and red colors indicate the different field line systems.

03:56 UT. However, the former produced the WLF, whereas the latter did not. This indicates that the first-step reconnection must occur at a lower altitude than the second-step one. In this way, high energy particles generated in the first-step reconnection can be relatively easily bombarded in the lower atmosphere.

For the second-step magnetic reconnection, the erupting flux rope stretches the envelope field to form a current sheet. Magnetic reconnection in the current sheet produces the long phase after the WLF peak. The main peak of the microwave burst appears at about 03:56 UT, when there is no more WL emission excess. The $\mathrm{H} \alpha$ ribbons clearly developed after 03:56 UT. In particular, the soft X-ray peak appearing at 03:53 UT is 13 min later than the WLF time (around 03:40 UT). All these phenomena can only be explained by the second-step magnetic reconnection. This two-step magnetic reconnection scenario is similar to the tether-cutting model that has been applied to solar eruptions (Moore et al. 2001). We must point out that the two-step reconnection is an approximate classification. However, it is already adequate to point out the key differences between the magnetic reconnections in the core and envelope fields.
Acknowledgements. We warmly thank the referee for advice that improved our paper in many ways. We also thank Dr. Yuying Liu, who acquired the radio data, Dr. Yang Liu, who provided the SDO/HMI data, and Dr. Junwei Zhao, who provided the helioseismic wave information. This work was supported by the National Natural Science Foundation of China (NSFC) under the grant numbers 10878002, 10610099, 10933003, 10673004 and 11103009, as well as a grant from the 973 project $2011 \mathrm{CB} 811402$.

\section{References}

Carrington, R. C. 1859, MNRAS, 20, 13

Chen, Q. R., \& Ding, M. D. 2005, ApJ, 618, 537

Chen, P.-F., Fang, C., \& Ding, M.-D. D. 2001, Chin. J. Astron. Astrophys., 1, 176

Ding, M. D., Fang, C., \& Yun, H. S. 1999, ApJ, 512, 454

Ding, M. D., Liu, Y., Yeh, C.-T., \& Li, J. P. 2003, A\&A, 403, 1151

Fang, C., \& Ding, M. D. 1995, A\&AS, 110, 99

Fang, C., Chen, P. F., Ding, M. D., Dai, Y., \& Li, Z. 2012, in EAS Publ. Ser., 55, 349

Fletcher, L., \& Hudson, H. S. 2008, ApJ, 675, 1645

Fletcher, L., Hannah, I. G., Hudson, H. S., \& Metcalf, T. R. 2007, ApJ, 656, 1187

Gan, W. Q., Rieger, E., Zhang, H. Q., \& Fang, C. 1992, ApJ, 397, 694

Gary, G. A., \& Hagyard, M. J. 1990, Sol. Phys., 126, 21

Guo, Y., Ding, M. D., Schmieder, B., Démoulin, P., \& Li, H. 2012, ApJ, 746, 17

Handy, B. N., Acton, L. W., Kankelborg, C. C., et al. 1999, Sol. Phys., 187, 229

Hudson, H. S., Acton, L. W., Hirayama, T., \& Uchida, Y. 1992, PASJ, 44, L77

Hudson, H. S., Wolfson, C. J., \& Metcalf, T. R. 2006, Sol. Phys., 234, 79

Jess, D. B., Mathioudakis, M., Crockett, P. J., \& Keenan, F. P. 2008, ApJ, 688, L119

Jiang, R. L., Fang, C., \& Chen, P. F. 2010, ApJ, 710, 1387

Leka, K. D., Barnes, G., Crouch, A. D., et al. 2009, Sol. Phys., 260, 83

Lemen, J. R., Title, A. M., Akin, D. J., et al. 2012, Sol. Phys., 275, 17

Li, Y. P., \& Gan, W. Q. 2006, ApJ, 652, L61

Liu, Z., \& Beckers, J. M. 2001, Sol. Phys., 198, 197

Liu, Y., Ding, M. D., \& Fang, C. 2001, ApJ, 563, L169

Machado, M. E., Emslie, A. G., \& Avrett, E. H. 1989, Sol. Phys., 124, 303

Machado, M. E., Avrett, E. H., Falciani, R., et al. 1986, in The lower atmosphere of solar flares, ed. D. F. Neidig, 483

Matthews, S. A., van Driel-Gesztelyi, L., Hudson, H. S., \& Nitta, N. V. 2003, A\&A, 409, 1107

Metcalf, T. R., Canfield, R. C., Avrett, E. H., \& Metcalf, F. T. 1990, ApJ, 350, 463

Moore, R. L., Sterling, A. C., Hudson, H. S., \& Lemen, J. R. 2001, ApJ, 552, 833

Neidig, D. F. 1989, Sol. Phys., 121, 261

Neidig, D. F., Wiborg, P. H., \& Gilliam, L. B. 1993, Sol. Phys., 144, 169

Tsuneta, S., Ichimoto, K., Katsukawa, Y., et al. 2008, Sol. Phys., 249, 167

Wang, H.-M. 2009, Res. Astron. Astrophys., 9, 127

Wheatland, M. S., Sturrock, P. A., \& Roumeliotis, G. 2000, ApJ, 540, 1150

Wiegelmann, T. 2004, Sol. Phys., 219, 87

Wiegelmann, T., Inhester, B., \& Sakurai, T. 2006, Sol. Phys., 233, 215

Xu, Y., Cao, W., Liu, C., et al. 2004, ApJ, 607, L131 